\title{
Research on Automotive Low-Frequency Electromagnetic Calibration System
}

\author{
Heqing Huang \\ IOT Department \\ Chongqing College of Electronic Engineering \\ Chongqing, China \\ hhqmail@126.com
}

\begin{abstract}
According to low-frequency electromagnetic compatibility problems of automotive electronic equipment, an automatic system based on 3-dimensional robot-arm was put forward and designed to detect electromagnetic field intensity. The recorded intensity of electromagnetic, and then implement three display functions of electromagnetic field in the light of visualization technology: 3D display of electromagnetic field distribution could show the strength and the trend of electromagnetic field. Extreme value display could pick up the maximum and minimum values. Critical scope display could draw the distribution of all points which range the users set. The design application field of whole system is very wide, and provides a feasible and practical reference for electromagnetic compatibility problem of large electrical equipment.
\end{abstract}

KEYWORDS-electromagnetic intensity; 3-dimensional; mechanical arm; auto detection;

\section{INTRODUCTION}

More and more electronic equipment have been used in car. The proportion of automotive electronics in vehicle cost keeps increasing in recent years, which has become an important sign to measure the level of automotive technology[1]. The electronic equipment improve the safety, comport and efficiency of the car, but on the other hand they also cause many problems in the design, testing and maintenance, such as mutual interference between electronic devices, electromagnetic interference of external environment on vehicle electronic equipment, and the electromagnetic compatibility problem is the most serious[2][6]. For example, the vehicle PEPS ( Passive Entry \& Passive Start ) low-frequency may not wake up the intelligent key to work due to the electromagnetic compatibility problem in car[2][3].The most automotive electronics manufacturers only pay attention on the product functions in the early design without consideration of electromagnetic compatibility, which will cause many EMC ( Electro Magnetic Compatibility ) testing prob- lems. Due to the lack of relevant automotive EMC design and rectification experience at present, the traditional method of electromagnetic field strength test adopts manual testing: for example, a tester uses a ruler to measure the coordinates of the test points, another tester holds the field strength tester to the test point and records the field strength. Such test procedure requires the corporation among several testers with time wast and high cost, and it is easy to introduce errors in the calibration of the test point coordinate and make it difficult to achieve the accuracy requirement[4][5]. So, it is important to develop a automatic test system with high accuracy for the automobile electromagnetic field measurement.

\section{SYSTEM STRUCTURE DESIGN}

\section{A. System hardware platform}

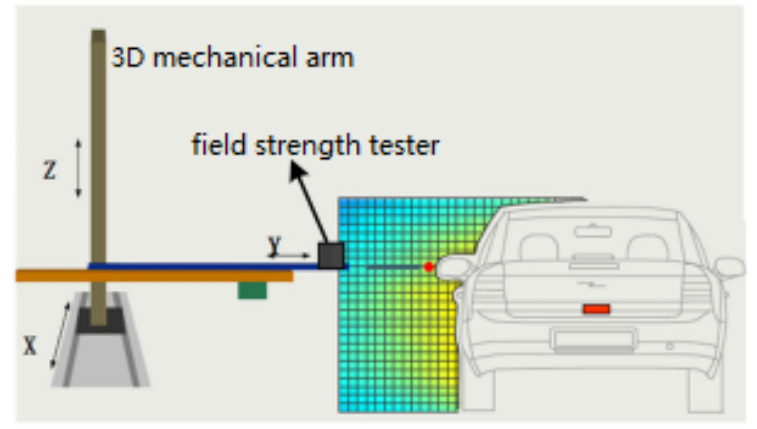

Fig. 1. Mechanical arm automatic moving diagram 
The automobile electromagnetic field automatic test system is an system integrated with hardware and software, which should be complied with some certain design principles[2]. The test system is mainly composed of computer, 3D program-controlled mechanical arm and field strength tester. The computer in system is the IPC ( Industrial Personal Computer) used widely. The 3D program-controlled arm is composed of controller, driver, three servo motors and three moving poles in $\mathrm{X}, \mathrm{Y}, \mathrm{Z}$ axis. The mechanical arm is connected with computer by motor controller, while the computer manage the three servo motors by this controller. The controller is responsible to generate the driving signal to each driver in $\mathrm{X}, \mathrm{Y}, \mathrm{Z}$ axis, and the driver make the pole to move by driving the corresponding motor. Each pole of mechanical arm can move in two directions with $0.001 \mathrm{~mm}$ step precision and can travel through the whole test area in 3D space. The figure 1 shows the effect diagram of mechanical arm automatic moving of the system, in which the driver of mechanical arm is programmed in PCI card and provide the users with dynamic library to develop. The application program interfaces of dynamic library can be used to fulfill mechanical arm initialization, parameters setting, speed configuration and moving poles control, etc. The collision rollback mechanism is adopted in mechanical arm to avoid the collision damage and make it more flexible, in which the mechanical arm will move back automatically when it is blocked by something. The field strength tester with width and height under $10 \mathrm{~cm}$ is fixed on the top of mechanical arm as shown in figure 1.

\section{B. System working procedure}

The system working procedure is composed of four parts: route table generation of testing-area, electromagnetic field strength data collection, result database generation of tested area and graphical display of electromagnetic field strength. After the tester used the IPC to initialize the testing area parameters by man-machine interface, the computer accesses the database to generate the route table of testing-area and drives the mechanical arm to travel the whole testing-area automatically under some certain moving rules at the same time. The field strength tester fixed on the top of the mechanical arm begins to record the electromagnetic strength values sent back by serial port. Then the IPC saves and analyses the data and displays the test positions and field strength values on the man-machine interface screen in real time, ant it generates result database for the tested area. At last, the part of graphic display of electromagnetic field strength shows the values in detail, which can be used to further analyze the distribution characteristics of the low frequency electromagnetic field strength in vehicle.

\section{SYSTEM IMPLEMENTATION}

\section{A. Route table generation design}

The low frequency electromagnetic field of car mainly distributes at the outside of left front door, around the hand-brake, back seats and trunk. Limited to the irregular car shape and the straight movement of mechanical arm along the $\mathrm{x}, \mathrm{y}, \mathrm{z}$ axis, it is impossible to complete the electromagnetic field measurement one time, which means it needs to measure many test fields to fulfill the whole-car's measurement. So it is necessary to design a reasonable route table for the mechanical arm to walk.

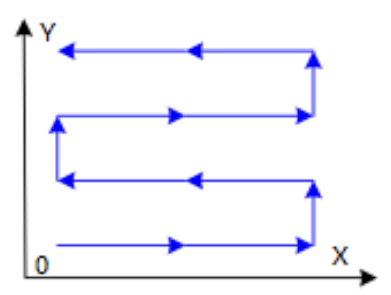

( a )

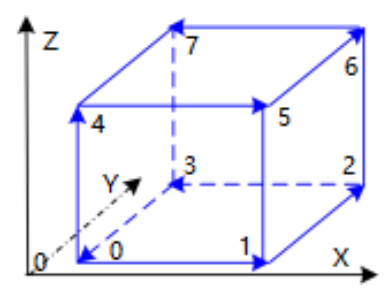

( b )
Fig 2. Traversing rule of testing area

A same coordinate origin is employed in this system for all of whole-car's electromagnetic field measurements, and an unified database is generated for each measurement procedure. As for some special test points around the car, it just need to control the mechanical arm to move to these test points to record the electromagnetic field values. A single test area can be approximately simulated into a rectangular test area, which can be traversed in $\mathrm{x}, \mathrm{y}$ and $\mathrm{z}$ axis order. First, the XOY plane will be traversed as shown in figure 2(a), in which the mechanical arm moves in the $\mathrm{x}$ axis until it completes the maximum steps then it moves one step in y axis and clear 
the steps of $x$ axis to zero, after that the mechanical arm will move again in $\mathrm{x}$ axis with opposite direction to the same maximum steps. Such action will be repeated until it gets the maximum steps of $y$ axis, then the steps of $x$ and $y$ axis will be cleared to zero. After finishing the measurement of XOY plane, the mechanical arm will move one step in $\mathrm{z}$ axis and test another XOY plane with the same rule. The moving rule of mechanical arm can be configured as an order of line-plane-stereo to complete electromagnetic field measurement of rectangle testing area. The testing area as shown as figure 2(b) can be traversed with 0-1-2-3-4-5-6-7 order. The coordinates of points 0 and 6 in rectangle diagonal can be used to define the position of testing area and generate the route table.

As for moving from a tested area to a testing area, it is shown in figure 3 that the mechanical arm may not be long enough to reach the testing point at the situation of moving from the outside of left front door of car ( V2 ) to the upside of trunk ( V3 ). Because the mechanical arm is fixed, so the it needs to adjust the car direction to complete the measurement of whole car. As a result, the spatial coordinate of testing area V3 will be changed relative to the origin coordinate of mechanical arm. As is shown in figure that the coordinate $(\mathrm{x}, \mathrm{y})$ of one testing point in testing area V3 rotated around the center point ( $\mathrm{x} 0, \mathrm{y} 0$ ) by angle $\theta$ will be mapped into the coordinate ( $\mathrm{x}^{\prime}$, $\left.y^{\prime}\right)$, so the coordinate mapping relationship is as following:

$$
\left\{\begin{array}{l}
x^{\prime}=\left(x-x_{0}\right) \cos \theta-\left(y-y_{0}\right) \sin \theta+x_{0} \\
y^{\prime}=\left(x-x_{0}\right) \sin \theta+\left(y-y_{0}\right) \cos \theta+y_{0}
\end{array}\right.
$$

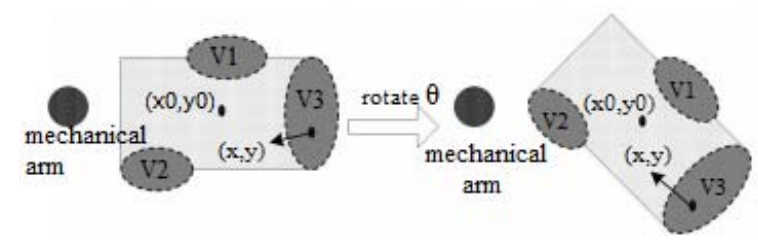

Fig 3. Rotating horizontally $\theta$ degree of car model

In order to simplify the calculation, the system set the center point $(\mathrm{x} 0, \mathrm{y} 0)$ of car as the coordinate origin point $(0,0)$ of the whole testing area of car, and the rotate degree is usually selected as 900,1800 , or 2700 . The work of coordinate transformation will be automatically done during the generation of measurement route table.

\section{B. Display design of electromagnetic field distribution}

The display of electromagnetic field distribution is mainly responsible for analysis and display of the electromagnetic field values collected by the electromagnetic field tester. It is used to research the problem of vehicle low frequency electromagnetic compatibility and also can provide the engineers with the design and rectification experience of automobile EMC solution. Three main functions are fulfilled in this system: distribution display of electromagnetic field, maximum and minimum values display of electromagnetic field, range display of electromagnetic field. The distribution display of electromagnetic field means reading the electromagnetic field value and coordinate of each test point from the result database of tested area and displaying all the test points on the visual interface. The color of each test point can be changed with the value of electromagnetic field, so it can reflect the distribution trend of electromagnetic field in the whole test area. The function of maximum and minimum values display is to read the electromagnetic field values of some tested area from the database, and it shows the maximum and minimum values and their positions in the visual interface. The range display of electromagnetic field is to show the distribution of all the test points within some certain value range set up by the user. As is discussed above, the distribution display of electromagnetic field can help to make an intuitive and accurate judgment of the distribution of electromagnetic field around car, which will be an important basis for the reach of electromagnetic field distribution of automotive electronic equipment.

\section{EXPERIMENTAL RESULT}

In order to verify the feasibility and accuracy of this testing system, an experiment was carried out in a EMC lab of a automobile company to verify the hardware, software and the whole logical processing. According to the experimental result, this system can accurately control the walk speed, direction and stop time of the mechanical arm, which can move accurately along the rectangle testing area. The electromagnetic field tester can get the accurate field strength value of the testing point and send it back to the IPC through the serial port. In order to verify the walking accuracy of the mechanical arm, the 
system select a range of testing area with $0.2 \mathrm{~m}^{*} 0.5 \mathrm{~m} * 1 \mathrm{~m}$ at random. The walk step is set with $0.1 \mathrm{~m}$, which means the mechanical arm will traverse 100 testing points. electromagnetic field tester will be transferred back to computer and shown timely on the interface screen with the position of testing point and its strength value. After that, the mechanical arm will continue to walk when it receives the command signal sent by computer.

The software testing mainly measures the electromagnetic field strength of the four areas V1-V4 around the car as shown in figure 3 with the $0.1 \mathrm{~m}$ walk step of mechanical arm. The experiment find out that this system can accurately generate the route table of the area to be tested and the result database of tested area, and it will automatically finish the area coordinate transformation after the car body transformation. At last, the system can also succeed to display the

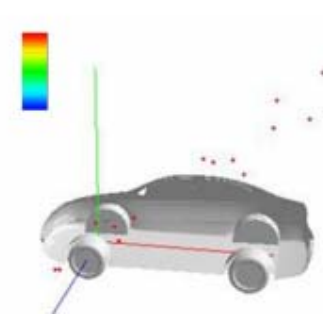

(a)

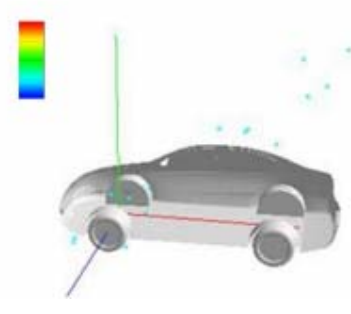

(b)
Fig 4. The display of extremum value in electromagnetic field

The figure 5 clearly shows the distribution of electromagnetic field about the four areas around the car. It means the greater value of electromagnetic field strength with the darker color, and the EMC problem is more serious. The positions of maximum values of electromagnetic field strength in four tested areas are shown in figure 4(a), and the positions of minimum values are shown in figure 4(b). All the points with the same extremum values can be displayed at the same time. The display of extremum values can help the engineers to find out the extremum values of electromagnetic field in certain range quickly. The EMC problem will occur when the strength of electromagnetic field reaches to a certain value in practice. electromagnetic field distribution at the same time, which will provide a basis for the reach and analysis of the electromagnetic field distribution characteristics of automotive electronic equipment. The electromagnetic field distribution of the experiment is shown in figure 5 .

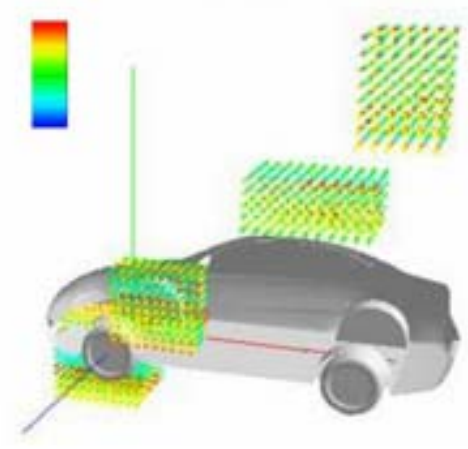

Fig 5. The distribution of electromagnetic field

\section{CONCLUSION}

The research of EMC problem is very important to the development of automotive electronic equipment. This paper provides an automatic electromagnetic detection system based on 3D mechanical arm usage. The technology of man-machine interaction is deployed in this system to fulfill the testing area initialization, display of electromagnetic field distribution, and the automatic walk of mechanical arm. This system with intelligence can solve the problems of traditional measuring method, such as time wasting, poor measurement accuracy, strong interference and poor operability.

\section{REFERENCES}

[1] Qing Hao, Jianpin Ou, Wenqiang Chen. Introduction of Keyless Start System[J]. Automotive electronic equipment. 2011(6);132-139.

[2] Xiaoping Zeng, Dongpin Xiao. Analysis of EMC on Automatic PEPS System[C]. National EMC conference.2012:48-56.

[3] $\mathrm{Xu} \mathrm{Li}$. The Analysis of EMC requirements on Automobile[J[. Electronic Quality.2002:52-56.

[4] Jiang X, Deng J, Liu L. Automobile PEPS Low-Frequency Automatic Calibration system's Accomplishment[C]. Proceedings of the International Conference on Information Engineering and Application (IEA) 2012:151-158.

[5] Jeffrey Travis, Jim Kring. LabVIEW for Everyone: Graphical Programming Made Easy and Fun, Third Edition[M]. America: Prentice Hall,2006.

[6] Qingshen Yang. The Research and Application of 3D Terrain Visualization based on OpenGL[D]. SiChuang University, 2008(5):22-28. 